\title{
Optimized Routing for People with Permanent or Temporary Mobility Disability: a Case Study in Viana do Castelo
}

\author{
David Verde \\ Instituto Politécnico de Viana do Castelo \\ Viana do Castelo, Portugal \\ davidverde@ipvc.pt
}

\author{
Sara Paiva \\ $A D i T-L A B$ \\ Instituto Politécnico de Viana do Castelo \\ Viana do Castelo, Portugal \\ sara.paiva@estg.ipvc.pt
}

\author{
Tânia Silva \\ Instituto Politécnico de Viana do Castelo \\ Viana do Castelo, Portugal \\ stania@ipvc.pt
}

\author{
Ana I. Pereira \\ CeDRI \\ Instituto Politécnico de Bragança \\ Bragança - 5300-253, Portugal \\ apereira@ipb.pt
}

\begin{abstract}
Nowadays, it is already common to have apps to assist citizens in their mobility within a city. However, apps are usually designed for the general citizen and do not include the specificity of people with reduced mobility, temporarily or permanently, such as visually impaired people, autistic people, people in wheelchairs, among others segments. This paper illustrates a case study carried out in the city of Viana do Castelo, in Portugal, where the streets of the historic center of the city were classified in a Geographic Information System (GIS) by the City Council together with the institutions that represent each one of the considered segments. Based on this classification, the Viana+Acessível app was developed, which is about to be made available free of charge to all citizens, and which recommends to each user the optimum route from a source to a destination, taking into account his segment. For example, visually impaired people should avoid streets where emergency vehicles can circulate and autistic people should preferably avoid streets with loud noises, among other conditions. The A-Star Algorithm and Dijkstra Algorithm were used in the app to identify the optimum route. A comparative study was made concluding that both strategies identify the optimum route and A-Star method obtains the optimum solution in a faster time. An evaluation of the app was also made in terms of its effectiveness and usability.
\end{abstract}

Index Terms-Inclusive Mobility; Routing Algorithms, Disabled People.

\section{INTRODUCTION}

The advent of smart cities, which we have witnessed as a result of technological advances in the last decades, combined with the emergence of concepts such as Big Data, Artificial Intelligence and Internet of Things as well as the integration of all these concepts and systems, makes it possible today to aggregate various data to give a more effective response to different domains that appear in cities in order to promote the increasingly relevant aspect of sustainability.

As a result of the demographic growth that we are witnessing, mobility is becoming a fundamental aspect in cities [1].
There are several challenges, from pedestrian movement and navigation in the center of cities to the use of the transport network for more distant travel, taking into account that there must be a rapid adaptation to the constant changes in the level of infrastructure that occur in cities frequently [2]. Urban orientation is intrinsically linked to mobility and refers to the ability of a citizen to know at every moment exactly where he is, whether or not he knows the city, and to be able to get directions to a desired location [3]. There are many apps to help people in this regard, but if we consider the segment of people with reduced mobility on a temporary or permanent basis, many other challenges arise and that are not always considered. As an example, visually impaired people should not, as a rule, pass in front of places with emergency services such as firefighters or hospitals, as they are places that represent great danger due to the possibility of sudden exits of vehicles. Another example is the segment of autistic people who, preferably, prefer to avoid streets with a lot of noise.

The United Nations agenda for 2030 [4] defines 17 Sustainable Development Goals (SGDs) where the aspect of inclusive mobility is highlighted so that people with reduced mobility can have access to all transport services with equal access level, and can also access places of leisure and public spaces in general.

This paper illustrates a case study carried out in the city of Viana do Castelo, in Portugal, in partnership with the City Council and which represents a continuation of previous work carried out in the field of mobility [5]. The main goal is to improve accessibility in the city for various people with reduced mobility (including visually impaired people [6], autistic people, deaf people, people in wheelchairs, elderly and pregnant people or people with children) in order to recommend them the optimal and most suitable route from their current location to an intended destination. The system 
is based on a Geographic Information System (GIS) that has an annotation of the various streets of the historic center of the city with regard to their suitability for each segment. At this moment there is an optimization only for the segments of visually impaired people and autistic people, which required a specific work with three associations of the city that represent these segments. A mobile application was developed with the main goal of recommending the most appropriate route to navigate to a given desired location, taking into account the user's segment, through a routing algorithm based on the AStar method.

The paper is organized as follows. Section II presents the related works associated with the study thematic work. The system overview describing the architecture proposed, routing strategies, data characterization and prototype version are presented in Section III. Section IV describes the prototype evaluation in terms of routing solution, effectiveness and usability. Last section concludes the paper by presenting the main conclusions and future work.

\section{RELATED WORKS}

The proliferation of the use of smartphones and mobile applications allowed its use in several domains, including the mobility of cities, route planning and assistance in the use of the transport network. An example of use is mentioned in [7], where authors focus on people with disabilities, and which are often not considered in the mobility solutions developed. The study presents an application for android that provides information about mobility in a city namely information regarding the schedules and routes made by each bus as well as the accessibility of each one. In the route recommendation, there is a concern to adapt it to the user's profile by choosing the transport with the best accessibility conditions.

In [8], authors present a framework that aims to assist people with reduced mobility to navigate from a source to a desired destination. The authors reinforce the idea that there are already many services that do route planning that take into account different types of transport, but that are mostly oriented towards the generality of people without taking into account the specific needs of people with reduced mobility. The presented framework is based on the creation of a graph with different levels of detail that can be modified in real time by users if they encounter any obstacle. The framework also takes into account, for the recommendation of the route, the walkways, crosswalks and obstacles that can be moved, such as terraces. The framework is based on OpenStreetMap (OSM) to manage the street graph model, OpenTripPlanner (OTP) to plan a route taking into account different restrictions and a mobile solution to detect obstacles and allow navigation in real time.

In [9], a systematic review of the literature is presented with regard to technological components that represent the building blocks of smart accessibility as well as a comparison of various technologies and existing case studies. A smart city architecture proposal is presented based on the modifications suggested by existing approaches that aim to centralize the accessibility of information in order to improve the mobility of people with reduced mobility. The proposed architecture gathers and stores disability-related geographical point of interests, provides customized routing services, uses opensource solutions for wheelchair access and makes the data available for open access.

Specific research for the segment of visually impaired people is also a focus for the scientific community. In [10], authors present three systems of guidance and mobility support for visually impaired people in order to help them navigate using different modes of transport in the city of Santiago, in Chile. The objective is to contribute so that these people are able to carry out their activities in a safe, autonomous and functional way. In [11], the authors identified necessary improvements in the transport network of the city of Copenhagen, so that they are more easily used by visually impaired people. The study identified the main challenges in accessing traffic information, collected the current accessibility conditions and evaluated accessible solutions in order to identify the most relevant and significant improvements. The authors concluded that announcements using audio should be used more, stricter regulation for bus operators should exist, accessibility of mobile applications should be extended and that an investment should be made on the implementation of an infrastructure using beacons in transport systems. In [12], authors present a routing algorithm adapted for visually impaired people on their pedestrian trips. The objective of the study is to present the best route from a source origin to a given destination, which can provide the visually impaired people with a sparse but helpful mental representation of the itinerary and surroundings. The solution presented was based on a set of brainstorming sessions with individual people and mobility and orientation instructors, which originated a Geographic Information System with annotation of different classes and objects. The final optimal route is selected using A-Star and Dijkstra algorithms. A-Star algorithm [13] and Dijkstra algorithms [14] are well known routing methods that are used in a large number of applications, namely vehicle routing problems, robot path planning problem, network optimization, transportation, logistics, among others [15], [16].

The present work is distinguished by its focus on several distinct segments of conditioned mobility and by the fact that it allows optimized routes for each one, using a GIS fed by information from the technicians of each institution, who know in detail the specifics and mobility needs of each segment.

\section{SYSTEM OVERVIEW}

This section describes the architecture of the system as well as its various components and algorithm, as well as the prototype app from the user's point of view.

\section{A. Architecture}

The system architecture consists of a mobile application, a routing algorithm and a GIS. As illustrated in Figure 1, the user interacts with the mobile application, indicating the location to which he intend to go (end point), and their current 
location is considered as the origin (start point). Based on these inputs, the algorithm calculates the most optimized route (which corresponds to the route with the least weight) and presents the route to the user on the map, using a color scheme that represents the suitability of the route, since different sections of the route can have different levels of suitability.

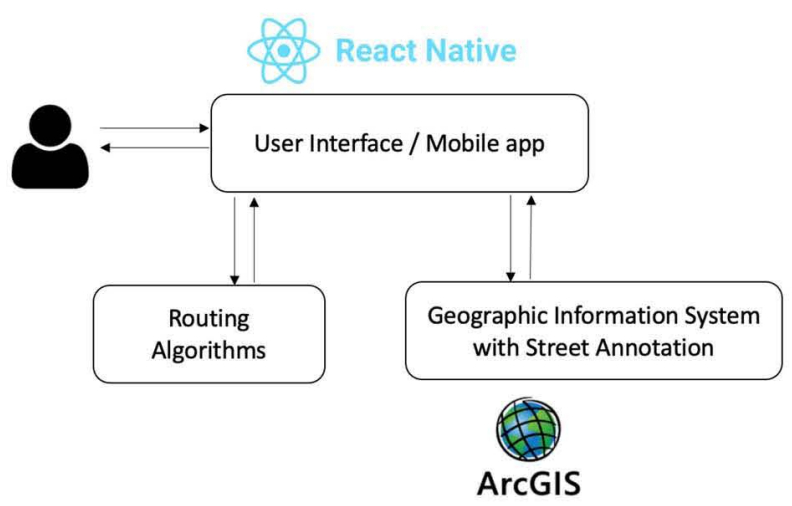

Fig. 1: System's architecture composed of a mobile app, a routing algorithm and a Geographic Information System

\section{B. Routing Algorithms}

In this work, A-Star and Dijkstra algorithms were used to identify the best solution from the start point to the end point selected by the user. A-Star heuristic algorithm is based on the combination of heuristic searching and searching based on the shortest path [13], [16]. A-Star algorithm is a search algorithm with information of all possible paths, keeping information that helps it prioritize its search. Dijkstra algorithm is a deterministic classical algorithm for solving the shortest path problem, commonly used to solve shortest-path tree and based on shortest path search [14].

\section{Data Characterization}

The routing algorithm operates on the data obtained from the GIS, managed by the City Council, which was built through the interaction with two institutions of visually impaired people (ACAPO and Íris Inclusiva) and an institution of autistic people (AMA). Together, they categorized the streets of the historic center into five levels, from exclusion to highly recommended. The exclusion level exists because there are streets that must be eliminated from the recommended route, even if this represents an increased time for commuting, due to the danger it implies for users. An an example of the categorization of GIS for the autistic segment is shown in Figure 2. The different colors of each segment represent different levels of suitability. The black color represents segments that should be excluded from the recommended routes and the yellow streets the most suitable. All other colors represent intermediary levels of suitability.

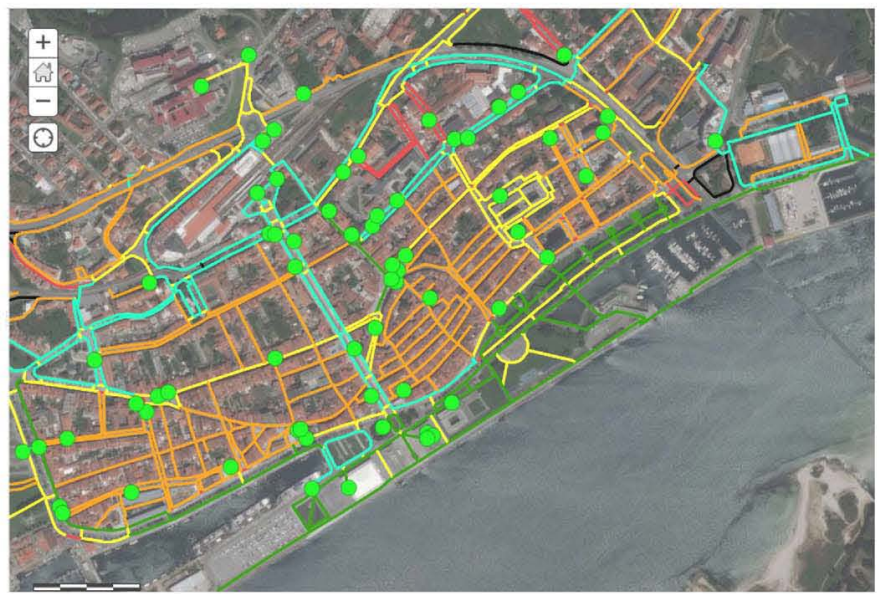

Fig. 2: GIS with the characterization of the streets for the autistic segment.

\section{Viana+Acessivel Application}

The main purpose of the developed mobile application is to recommend the most suitable route for a given destination, depending on the user's segment. For this, the first screen of the application allows the user to choose their condition from among six possibilities: people with null or reduced vision, people in wheelchairs, people with deafness, people with autism spectrum disorder, pregnant women, people with strollers baby or small children or old people. After this initial choice, the user sees the screen shown at Figure 4 (a) that has four main features, accessible via the buttons at the bottom of the screen. The first button on the left side allows the visualization on the map of several places of interest in the city referring to five categories: culture, health, public services, transport or tourism. The third button shows the parking places for people with disabilities and the fourth button shows taxi points. In any of these screens, the user can click on the marker to see the information about the place and also an optimized route from the current location to the selected place, being presented with the screen represented in Figure 4 (c). On this screen, it is possible to see the suitability of each part of the route, taking into account the color scheme shown at the bottom of the screen. Finally, the second button on the screen Figure 4 (b) allows viewing the complete route network for the user's segment, where each street is identified according to its suitability, also according to the color scheme in the bottom of the screen.

It is important to mention that, as this application is optimized for the segment of visually impaired people, it is not intended to be used directly by them, but by someone who accompanies them and who can thus know the most suitable route to use when traveling with a visually impaired person. On the other hand, depending on the type of blindness, the person may still have a percentage of vision that allows them to use the app autonomously. 


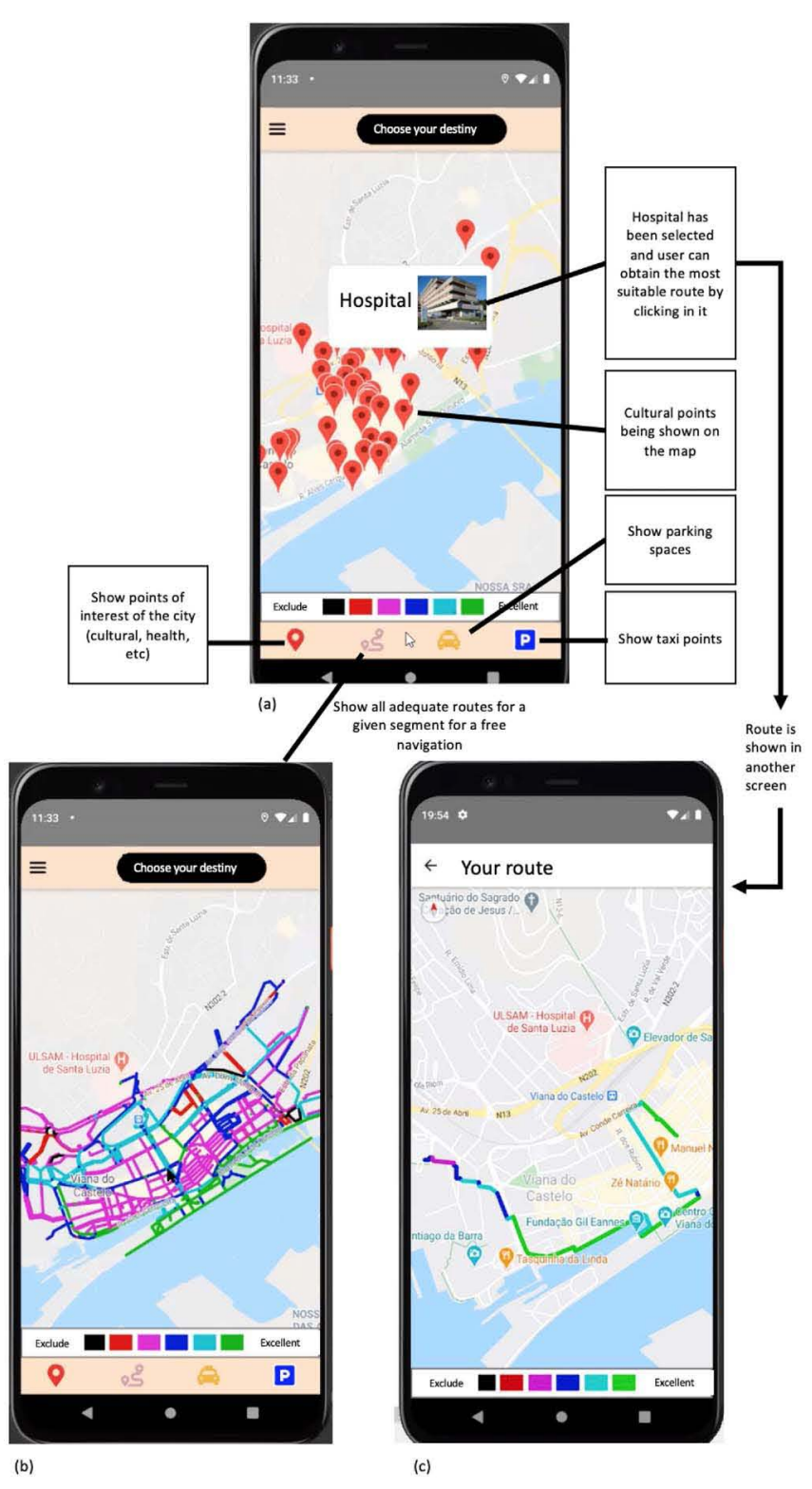

Fig. 3: Prototype showing the main functionalities of the app as well as the most suitable route on the map.

\section{EVALUATION}

This section presents different techniques to evaluate the routing solution as well the application perform.

\section{A. Algorithms comparison}

A-Star and Dijkstra algorithms run under React Native and Javascript language environment and six different objective paths were used to analyse the performance of the different algorithms. The main characteristics of the study paths are presented in Table I where it is presented the Start and End points of each path. The notation of $S_{i}, M_{i}$ and $L_{i}$, for $i=1,2$, represent short, medium and long length path, respectively.

Both methods obtain the same optimum path with small different on the execution time. To evaluate the time perfor-

\begin{tabular}{c|c|c} 
& Start Point & End Point \\
\hline Path $S_{1}$ & Hospital Sta. Luzia & Interface \\
Path $S_{2}$ & Jardim da Marina & Farmácia Manso \\
Path $M_{1}$ & Avenida de Cabo Verde & CTT Praça Alto Minho \\
Path $M_{2}$ & Avenida do Atlântico & Rua de Monserrate \\
Path $L_{1}$ & Rua Penedo & Farmácia São Bento \\
Path $L_{2}$ & Rua Frei Bartolomeu dos Mártires & CTT Praça Alto Minho
\end{tabular}

TABLE I: Study paths characteristics

mance of each algorithm, 100 runs were done and the Tables II and III present the average algorithm execution time (AT) for each path in ms, the standard deviation (SD), the median value (MedT), the maximum execution time (MaxT) and minimum execution time (minT) for A-Star and Dijkstra algorithms.

\begin{tabular}{|c|c|c|c|c|c|}
\hline & AT & SD & MedT & MaxT & minT \\
\hline Path $S_{1}$ & 42.9 & 1.82 & 42 & 79 & 40 \\
\hline Path $S_{2}$ & 50.6 & 2.46 & 50 & 118 & 47 \\
\hline Path $M_{1}$ & 86.7 & 1.99 & 86 & 120 & 83 \\
\hline Path $M_{2}$ & 54.4 & 1.91 & 53 & 97 & 51 \\
\hline Path $L_{1}$ & 87.9 & 1.81 & 87 & 128 & 84 \\
\hline Path $L_{2}$ & 86.1 & 2.05 & 85 & 124 & 83 \\
\hline
\end{tabular}

TABLE II: Execution time values for A-Star algorithm

Using the A-Star algorithm it was verified that the amplitude of execution time varies between 71 to $37 \mathrm{~ms}$ considering the maximum and minimum execution times. The Path $S_{2}$ was the path with more time variation $(71 \mathrm{~ms})$.

Table III presents the results obtained by Dijkstra method.

\begin{tabular}{|c|c|c|c|c|c|}
\hline & AT & SD & MedT & MaxT & minT \\
\hline Path $S_{1}$ & 44.4 & 1.94 & 43 & 95 & 42 \\
\hline Path $S_{2}$ & 50.4 & 1.92 & 49 & 78 & 48 \\
\hline Path $M_{1}$ & 88.1 & 1.72 & 87 & 122 & 85 \\
\hline Path $M_{2}$ & 53.7 & 1.95 & 53 & 95 & 51 \\
\hline Path $L_{1}$ & 89.9 & 2.01 & 89 & 138 & 86 \\
\hline Path $L_{2}$ & 88.1 & 2.24 & 87 & 134 & 84 \\
\hline
\end{tabular}

TABLE III: Execution time values for Dijkstra algorithm

Using Dijkstra algorithm, the Path $L_{1}$ was the path with more execution time variation (difference between 95 and 42 $\mathrm{ms})$. Analysing both tables, it is possible to observe that, in average, the optimum path was obtaining on less $100 \mathrm{~ms}$ and, in general, the A-Star algorithm obtained the optimum path in less time when compared with Dijkstra algorithm results.

\section{B. Effectiveness}

In order to validate that the recommended routes are effectively adequate to the users' needs, several routes were tested, the main form of validation being confirmation by the institutions involved. An institution in the segment of visually impaired people and another in the segment of autistic people sent three of the routes most made by users in their daily lives. The route calculated by the app was sent for their validation. This validation work led to the confirmation that the app is calculating the route correctly according to the segment classification carried out jointly by the institutions and the City Council.

An example of how that validation process was done is presented next. For simplicity reasons, we present only one of 
the evaluated routes, for the autism spectrum disorder segment. Figure 4 shows the optimized route on the left and the fastest route on the right, the difference being noticeable. On the left side of Figure 4, it is possible to see that the optimized route is mostly constituted by green (Excellent) and cyan segments, just being applied the dark blue in two crosswalks. On the right side of Figure 4, the algorithm does not have in consideration the disability of the user, but the time that takes to go through each segment, what leads to less suitable paths. The fastest route has a total length of 1289 meters and takes 17 minutes to cover, while the most suitable route takes 26 minutes to cover 2153 meters. This route is intended for users with autism spectrum disorder. As such, classification of the streets was made to avoid crowds, very loud noises and movement of cars that disturb the autonomy of these users. In the fastest path, the black segment is classified as 'Exclude' (and therefore not included in the optimized route) because it is a place with a lot of movement of vehicles, loud noises and low visibility. The purple segments are classified as 'Avoid' because they represent streets with buildings in both sides and a high movement of people and vehicles. The blue segments represent crosswalks with some movement of vehicles, less danger that the black segment, but that require some attention from the user.
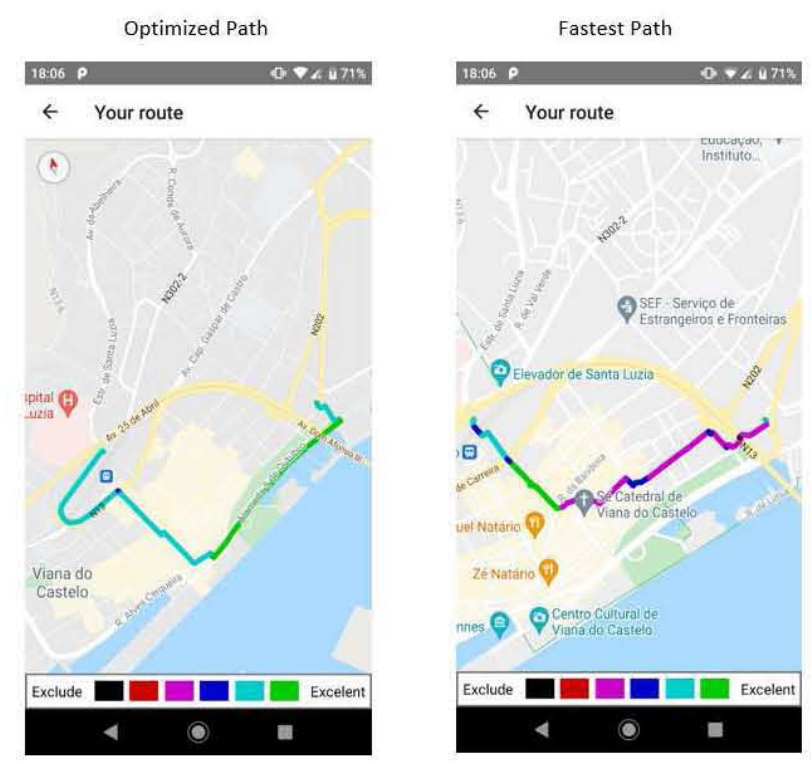

Fig. 4: Validation process - optimized versus fastest route for the Autism Spectrum Disorder segment

\section{Usability}

The developed prototype was subjected to a usability test that was initially expected to be carried out by visually impaired people and autistic people, which was not possible due to the current pandemic situation. The prototype was thus evaluated by two technicians who accompany the autistic segment and by one technician who accompanies visually impaired people.

The questions used, shown in Table IV, were based on the System Usability Scale [17], with 10 questions, to which users have 5 answer options ('Strongly Agree', 'Agree', 'Neither Agree nor Disagree', 'Disagree' or 'Strongly Disagree') that correspond to numeric values from 1 to 5 .

The final score used the methodology described in [18], described also below:

- Add all the scores for the odd numbered questions (1, 3, $5,7,9)$ together and subtract 5 from this;

- Add all the scores for the even numbered questions together $(2,4,6,8,10)$ and subtract this number from 25;

- Add the scores calculated in steps 1 and 2 together and multiply by 2.5 ;

- Round the number calculated in step 3 to nearest whole number to get the final SUS score.

A score of 68 is considered to be the average. The obtained scores are presented in $\mathrm{V}$ and represent an average final score of 83 , which represents a very positive assessment on the part of the technicians who accompany people from two representative segments of the solution and who know the real needs of users. It should be noted that there is a much higher assessment on the part of technicians in the autistic segment and an assessment on the threshold of the average by the visually impaired segment, so it is necessary, as soon as possible, to make usability tests with the users themselves in order to obtain their perception and be able to further improve the usability of the app.

TABLE IV: List of SUS Questions

\begin{tabular}{|c|c|}
\hline Question 1 & I would like to use the system frequently. \\
\hline Question 2 & The system was unnecessary complex. \\
\hline Question 3 & The system is easy to use. \\
\hline Question 4 & $\begin{array}{l}\text { The support of a technical person was needed to } \\
\text { use the system. }\end{array}$ \\
\hline Question 5 & $\begin{array}{l}\text { The various functions of the system were well } \\
\text { integrated. }\end{array}$ \\
\hline Question 6 & There was too much inconsistency in the system. \\
\hline Question 7 & $\begin{array}{l}\text { People would learn to use the system very } \\
\text { quickly. }\end{array}$ \\
\hline Question 8 & The system was very cumbersome to use. \\
\hline Question 9 & I am very confident using the system. \\
\hline Question 10 & $\begin{array}{l}\text { I needed to learn a lot of things before I could } \\
\text { get on with the system. }\end{array}$ \\
\hline
\end{tabular}

\section{CONCLUSIONS AND FUTURE WORK}

In this paper, a case study was presented in the city of Viana do Castelo about inclusive mobility, more specifically the development of the Viana+Acessivel app that recommends optimized routes, using the A-Star and Dijkstra algorithms, for people with reduced mobility, supported by a GIS that has the characterization of the city's streets in terms of suitability for each considered segment, with optimization at this moment for the visually impaired and autistic people. The GIS was 
TABLE V: SUS Scores for the four Testers

\begin{tabular}{|c|c|c|c|}
\hline & Tester 1 (VIP) & Tester 2 (Autism) & Tester 3 (Autism) \\
\hline Question 1 & 5 & 5 & 5 \\
\hline Question 2 & 1 & 1 & 1 \\
\hline Question 3 & 3 & 5 & 5 \\
\hline Question 4 & 4 & 1 & 1 \\
\hline Question 5 & 4 & 5 & 5 \\
\hline Question 6 & 2 & 1 & 1 \\
\hline Question 7 & 4 & 5 & 5 \\
\hline Question 8 & 2 & 1 & 1 \\
\hline Question 9 & 4 & 5 & 5 \\
\hline Question 10 & 4 & 5 & 5 \\
\hline Score & 68 & 90 & 90 \\
\hline
\end{tabular}

created by the City Council together with the institutions that support each segment, stating this as a project that arises from the synergy of several stakeholders.

The paper features an evaluation section in which the performance of A-Star and Dijkstra algorithms were compared and also the effectiveness and usability of the app. The optimum path was obtained in all study paths and, usually, A-Star algorithm identify the optimum solution faster than Dijkstra algorithm. From the tests carried out with the stakeholders, it became evident that this is a continuous work as the creation or elimination of a new crosswalk or a walkway can significantly change the suitability of the streets for a given segment, so maintaining the correct classification of the streets in the GIS over time presents itself as the biggest challenge, but also a fundamental piece so the app's algorithm is able to recommend routes appropriately.

The future work involves extending the recommendation of routes to include longer paths that involve electric buses in the city and even those that travel to the periphery, favoring electric buses for a greater contribution for sustainable mobility and a multiobjective approach also will be considered in future works.

\section{REFERENCES}

[1] V. K. Giduthuri, "Sustainable urban mobility: Challenges, initiatives and planning," in Current Urban Studies, no. 3, 2015, pp. 261-265.

[2] M. Bezbradica and H. J. Ruskin, "Understanding urban mobility and pedestrian movement, smart urban development," in IntechOpen, 2019.

[3] A. Riazi, F. Riazi, R. Yoosfi, and F. Bahmeei, "Outdoor difficulties experienced by a group of visually impaired iranian people," Journal of Current Ophthalmology, vol. 28 , no. 2, pp. 85-90, 2016. [Online]. Available: https://www.sciencedirect.com/science/article/pii/S245223251530055X

[4] U. Nations, "Transforming our world: the 2030 agenda for sustainable development," 2020, last accessed on March 31st 2021. [Online]. Available: https://sustainabledevelopment.un.org/post2015/transformingourworld

[5] D. Brito, T. Viana, D. Lourenço, and S. Paiva, "A mobile solution to help visually impaired people in public transports and in pedestrian walks," in Journal of Sustainable Development and Planning, vol. 13, no. 2, 2018, pp. 281-293.

[6] S. Paiva and N. Gupta, Technologies and Systems to Improve Mobility of Visually Impaired People: A State of the Art. Cham: Springer International Publishing, 2020, pp. 105-123.

[7] S. Cabral, M. Mónica, J. Metrôlho, and F. Ribeiro, "App for more inclusive urban mobility a prototype in development," in 2019 14th Iberian Conference on Information Systems and Technologies (CISTI), 2019, pp. 1-4.
[8] A. Mancini and P. Zingaretti, "Point to point navigation for people with mobility impairments," in 2014 IEEE/ASME 10th International Conference on Mechatronic and Embedded Systems and Applications (MESA), 2014, pp. 1-6.

[9] Y. R. Panta, S. Azam, B. Shanmugam, K. C. Yeo, M. Jonkman, F. De Boer, and M. Alazab, "Improving accessibility for mobility impaired people in smart city using crowdsourcing," in 2019 Cybersecurity and Cyberforensics Conference (CCC), 2019, pp. 47-55.

[10] J. Sánchez, M. d. B. Campos, M. Espinoza, and L. B. Merabet, "Accessibility for people who are blind in public transportation systems," in Proceedings of the 2013 ACM Conference on Pervasive and Ubiquitous Computing Adjunct Publication, ser. UbiComp '13 Adjunct. New York, NY, USA: Association for Computing Machinery, 2013, p. 753-756. [Online]. Available: https://doi.org/10.1145/2494091.2496002

[11] J. C. Flynn, J. Komissar and A. Paul, "Improving accessibility of public transport information for the blind," Ph.D. dissertation, Worcester Polytechnic Institute, 2018.

[12] S. Kammoun, F. Dramas, B. Oriolaand, and C. Jouffrais, "Route selection algorithm for blind pedestrian," in ICCAS 2010, 2010, pp. 2223-2228.

[13] P. Hart, N. J. Nilsson, and B. Raphael, "A formal basis for the heuristic determination of minimum cost paths," IEEE Transactions on Systems, Science and Cybernetics SSC, vol. 4.

[14] E. W. Dijkstra, "A note on two problems in connection with graphs," Numerische Mathematik, vol. 1.

[15] Z. Zhang and Z. Zhao, "A multiple mobile robots path planning algorithm based on a-star and dijkstra algorithm," International Journal of Smart Home, vol. 8.

[16] S. Rabin, "A* speed optimizations," Game Programming Gems, pp. 272 287,2000 .

[17] J. Brooke, "Sus: A quick and dirty usability scale," Usability Eval. Ind., vol. 189, 111995.

[18] S. Hayes, S. Wang, and S. Djahel, "Personalized road networks routing with road safety consideration: A case study in manchester," in 2020 IEEE International Smart Cities Conference (ISC2), 2020, pp. 1-6. 